ISSN = 1980-993X - doi:10.4136/1980-993X
www.ambi-agua.net
E-mail: ambi-agua@agro.unitau.br
Tel.: (12) 3625-4212

\title{
Uso del modelado de nicho ecológico como una herramienta para predecir la distribución potencial de Microcystis sp (cianobacteria) en la Presa Hidroeléctrica de Aguamilpa, Nayarit, México
}

\author{
(http://dx.doi.org/10.4136/ambi-agua.607)
}

\author{
José L. Ibarra-Montoya ${ }^{1}$; Gabriel Rangel-Peraza ${ }^{1}$; Fernando A. González-Farias ${ }^{2}$; \\ José De Anda ${ }^{1}$; Enrique Martínez-Meyer ${ }^{3}$ y Humberto Macias-Cuellar ${ }^{4}$ \\ ${ }^{1}$ Centro de Investigación y Asistencia en Tecnología y Diseño del Estado de Jalisco, México \\ e-mail: jibarra@ecologia.unam.mx; jgrp2000@hotmail.com; janda@ ciatej.net.mx; \\ ${ }^{2}$ Instituto de Ciencias del Mar y Limnología, Universidad Nacional Autónoma de México \\ e-mail: gfarias@servidor.unam.mx; \\ ${ }^{3}$ Instituto de Biología, Universidad Nacional Autónoma de México \\ e-mail: emm@ibunam2.ibiologia.unam.mx; \\ ${ }^{4}$ Departamento de Investigación Acción Participativa y Planeación Territorial Comunitaria, \\ Chakaan Buulaan, A. C. \\ e-mail: maciascuellar@chakaan.org
}

\section{RESUMEN}

El modelado de nicho ecológico es una herramienta importante para la evaluación de la distribución espacial de especies terrestres, sin embargo, su aplicabilidad ha sido poco explorada en el medio acuático. Microcystis sp., es una especie de cianobacteria con frecuencia conocida por la producción de toxinas del tipo microcistinas, cuya ingestión en altas concentraciones ocasiona la muerte de animales, desde pequeñas aves hasta el ganado. Como cualquier grupo taxonómico, ésta cianobacteria presenta umbrales ambientales, es decir; un nicho ecológico idóneo que va a delimitar su distribución. El presente estudio se realizó en la Presa Hidroeléctrica Aguamilpa, un ecosistema artificial que entró en operación en 1994. En este sistema se evaluó la distribución potencial de Microcystis sp., mediante la generación de un modelo de predicción basado en el concepto de nicho ecológico MAXENT; empleando un Modelo Digital de Elevación en celdas de 100 m x 100 m (1 ha) de resolución espacial y once variables físicas, químicas y biológicas del agua. La elaboración de los mapas de distribución, se realizó con ArcMap 9.2 ${ }^{\circledR}$. Los resultados indican que Microcystis sp., se distribuye principalmente en la cuenca alta del afluente del río Huaynamota, tanto en el estiaje frío, como en el cálido. Sin embargo, existe una menor probabilidad de encontrarla en todo el sistema de la presa durante el estiaje frío, mientras que durante el estiaje cálido también se localiza en la confluencia de ambos ríos. Para la época de lluvias no se tienen reportes de la presencia de ésta cianobacteria. Esta especie, generalmente está asociada a procesos tróficos derivados de la presencia de contaminantes de origen antropogénico. Las actividades humanas en la cuenca (por ejemplo, agricultura tradicional, ganadería, actividades industriales) y los escurrimientos, han afectado la distribución de Microcystis sp., dados los efectos deletéreos de la contaminación. Se requerirá atención en las áreas específicas que se han identificado en este trabajo, así como medidas de manejo y restauración de las cuencas. También se documenta una interacción entre fósforo y nitrógeno que determina la distribución de Microcystis sp. El modelado de nicho ecológico es una herramienta adecuada para la evaluación de la distribución espacial de microalgas en ambientes dulceacuícolas.

Palabras clave: Microcystis sp.; cianobacteria; modelo de nicho ecológico; presas; MAXENT. 


\title{
Use of ecological niche modeling as a tool for predicting the potential distribution of Microcystis sp (cyanobacteria) in the Aguamilpa Dam, Nayarit, Mexico
}

\begin{abstract}
Ecological niche modeling is an important tool to evaluate the spatial distribution of terrestrial species, however, its applicability has been little explored in the aquatic environment. Microcystis sp., a species of cyanobacteria, is widely recognized for its ability to produce a group of toxins known as microcystins, which can cause death of animals as fish, birds and mammals depending on the amount of toxin absorbed. Like any taxonomic group, cyanobacteria has environmental thresholds, therefore, a suitable ecological niche will define their distribution. This study was conducted in Aguamilpa Hydroelectric Reservoir, an artificial ecosystem that started operations in 1994. In this system we evaluated the potential distribution of Microcystis sp., by generating a prediction model based on the concept of ecological niche MAXENT, using a Digital Elevation Model in cells of $100 \mathrm{~m}$ x $100 \mathrm{~m}$ (1 ha) spatial resolution and monitoring eleven physicochemical and biological variables and nutrients in water. The distribution maps were developed using ArcMap 9.2 ${ }^{\circledR}$. The results indicated that Microcystis sp., is distributed mainly in the upper tributary basin (Huaynamota basin) during the dry season. There was less chance to find cyanobacteria in the entire system during the cold dry season, while during the warm dry season cyanobacteria was recognized at the confluence of two rivers. During the rainfall season there were no reports of cyanobacteria presence. This species is often associated with arising trophic processes of anthropogenic origin; therefore, attention is required in specific areas that have been identified in this work to improve Aguamilpa's watershed management and restoration. It was also recognized the importance of phosphorus and nitrogen interaction, which determines the distribution of Microcystis sp., in the Aguamilpa Reservoir. The results of this study demonstrated that ecological niche modeling was a suitable tool to assess the spatial distribution of microalgae in freshwater environments.
\end{abstract}

Keywords: Micocystis sp.; cyanobacteria; ecological niche model; reservoir; MAXENT.

\section{Uso de modelagem de nicho ecológico como ferramenta para previsão da distribuição potencial de Microcystis sp (cyanobacteria), na Usina Hidrelétrica de Aguamilpa, Nayarit, México}

\section{RESUMO}

Modelagem de nicho ecológico é uma ferramenta importante para se avaliar a distribuição espacial das espécies terrestres, no entanto, sua aplicabilidade tem sido pouco explorada no ambiente aquático. Microcystis sp., uma espécie de cianobactérias, é amplamente reconhecida pela sua capacidade de produzir um grupo de toxinas conhecidas como microcistinas, o que pode causar a morte de animais como peixes, aves e mamíferos, dependendo da quantidade de toxina absorvida. Como qualquer grupo taxonômico, as cianobactérias têm limites ambientais, portanto, um nicho adequado ecológico define sua distribuição. Este estudo foi realizado no reservatório da usina hidroelétrica Aguamilpa, um ecossistema artificial que iniciou suas operações em 1994. Neste sistema, foi avaliada a distribuição potencial de Microcystis sp., para se gerar um modelo de previsão baseado no conceito do nicho ecológico MAXENT, utilizando um Modelo de Elevação Digital com 

predecir la distribución potencial de Microcystis sp (cianobacteria) en la Presa Hidroeléctrica de Aguamilpa, Nayarit, México. Ambi-Agua, Taubaté, v. 7, n. 1, p. 218-234, 2012. (http://dx.doi.org/10.4136/ambiagua.607)

células de 100 m x 100 m (1 ha) de resolução espacial e o monitoramento de 11 variáveis físico-químicas, biológicas e nutrientes na água. Os mapas de distribuição foram desenvolvidos utilizando o ArcMap 9.2 ${ }^{\circledR}$. Os resultados indicaram que a Microcystis sp. Distribui-se principalmente na parte superior da bacia afluente (bacia Huaynamota) durante a estação seca. Houve menos chances de se encontrar cianobactérias no sistema durante a estação fria e seca, enquanto que durante a estação quente e seca, cianobactérias foram reconhecidas na confluência de dois rios. Durante o período chuvoso não houve relatos da presença de cianobactérias. Esta espécie é frequentemente associada com processos tróficos decorrentes de origem antropogênica, portanto, atenção é necessária em áreas específicas que foram identificadas neste trabalho para melhorar a gestão e a restauração de bacias hidrográficas em Aguamilpa. Foi também reconhecida a importância da interação entre o fósforo e nitrogênio, na distribuição de Microcystis sp., no reservatório Aguamilpa. Os resultados deste estudo demonstraram que a modelagem de nicho ecológico foi uma ferramenta adequada para se avaliar a distribuição espacial de microalgas em ambientes de água doce.

Palavras-chave: Microcystis sp.; cyanobacteria; o modelo de nicho ecológico; reservatório; MAXENT.

\section{INTRODUCCIÓN}

El modelado de nicho ecológico (MNE) es probablemente el método más adecuado que actualmente existe para estimar la distribución geográfica real y potencial de las especies (Guisan y Thuiller, 2005). Este enfoque se utiliza cada vez más en la toma de decisiones en materia de conservación, restauración y contaminación ambiental (Pearce y Lindenmayer, 1998; Ferrier, 2002). A pesar de la probada eficacia de este método para la delimitación de las distribuciones geográficas de las especies terrestres, su aplicabilidad en el ámbito acuático ha sido poco explorada (Wiley et al., 2003). Esto es particularmente cierto para los ecosistemas de agua dulce, donde la escala geográfica es grande, y la información de los datos ambientales, para la mayor parte del mundo no es de calidad; esta información es necesaria para la generación de los modelos de nicho (Iguchi et al., 2004; McNyset, 2005). Tal es el caso de los ecosistemas limnológicos en México, donde los datos normalmente están incompletos y no se cuenta con bases de datos geográficos, regionales o nacionales, de las características físicas, químicas y biológicas de las aguas continentales.

La Presa Hidroeléctrica (P. H.) de Aguamilpa, se ubica en el borde sureste de la Sierra Madre Occidental, con la presencia de terrenos con relieves accidentados de origen volcánico. Sus características principales son dos sistemas de topoformas: la sierra alta, ubicada en la subcuenca del río Huaynamota, formada por altas mesetas interrumpidas de manera abrupta por cañadas; y la del tipo cañón, en la subcuenca Santiago-Aguamilpa, donde el relieve se conforma de cadenas montañosas de formas volcánicas escarpadas y cañones con perfiles en "V", que llegan a alcanzar una altura por arriba de los 1,000 m en algunos sitios (INEGI, 2006a).

En el embalse de la P. H. de Aguamilpa, se ha registrado una gran diversidad de especies de fitoplancton, que incluyen a Microcystis, Anabaena y Aulacoseira como los géneros más abundantes durante el estiaje cálido y algunas especies de Clorofitas durante el estiaje frío (Zamudio y González-Farias, 2009). Uno de los grupos más representativos de la comunidad de fitoplancton del embalse es el de las cianobacterias, entre las que se tiene a Microcystis sp., una especie que crece preferentemente en ambientes con alta concentración de nutrientes, principalmente fósforo y nitrógeno (Lampert y Sommer, 1997; Bernal-Brooks et al., 2003; 
Dávalos et al., 1989), por lo que es común encontrarla en cuerpos de agua eutrofizados (Roset et al., 2001), y puede producir potentes toxinas del tipo de las microcistinas, capaces de originar efectos agudos y crónicos en el hombre y en las diversas especies de animales (Cood et al., 1999).

A nivel mundial se tiene gran interés en el estudio del género Microcystis, debido a las numerosas intoxicaciones letales en diversas especies de fauna silvestre y doméstica, y en el número de casos de enfermedades causadas a los humanos por la exposición prolongada a las toxinas, situación que ha llamado la atención de la Organización Mundial de la Salud (OMS) de la sociedad en general y de la comunidad científica en particular (Chorus, 2001).

Dada la importancia de Microcystis sp., en este trabajo se presenta un análisis espacial, basado en un modelo de nicho ecológico que permite generar mapas predictivos de las zonas de presencia de esta especie en el embalse de Aguamilpa, en función de los parámetros ambientales, las variables físico-químicas y biológicas del agua y los parámetros hidrológicos. Se considera una excelente oportunidad para establecer estrategias que permitan conocer la distribución potencial de Microcystis sp., en el ecosistema acuático y contrastarlo con las actividades propias del uso de suelo y vegetación de las subcuencas.

\section{2. Área de Estudio}

La P. H. de Aguamilpa se localiza en la región central del estado de Nayarit, México, y comprende los municipios del Nayar, la Yesca, Santa María del Oro y Tepic. Está localizada entre los meridianos $104.39^{\circ}$ y $104.74^{\circ}$ de longitud oeste y los paralelos $21.51^{\circ}$ y $21.9^{\circ} \mathrm{de}$ latitud norte (Figura 1). Se ubica en el borde sureste de la Sierra Madre Occidental, con la presencia de terrenos con relieves accidentados de origen volcánico. Presenta dos ramas, una de $50 \mathrm{~km}$ de largo y la otra de $20 \mathrm{~km}$ aproximadamente, que son los cauces originales de los ríos Santiago y Huaynamota, respectivamente. Exhibe además un cuerpo de agua amplio e irregular que se forma desde la cortina de la presa hasta el punto de confluencia de ambas ramas (Figura 1).

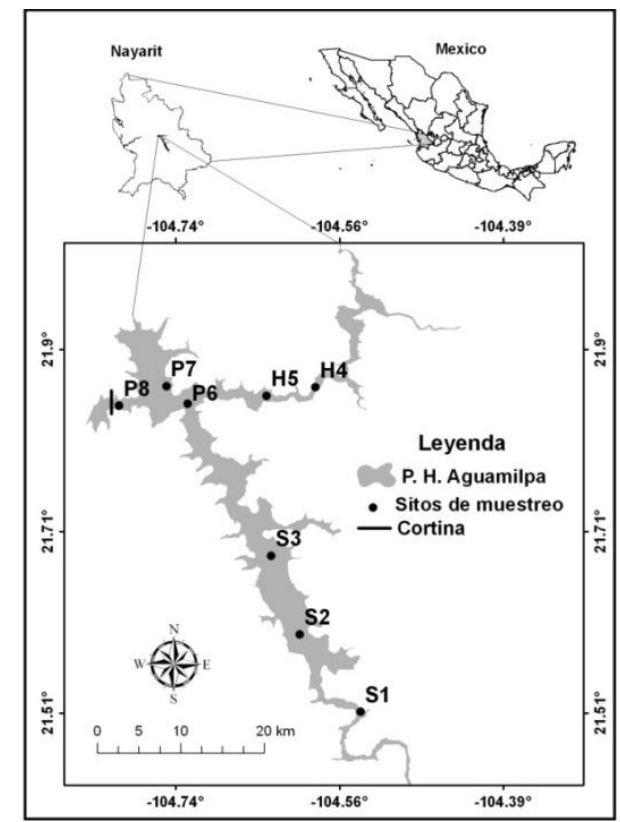

Figura 1. Mapa de localización del área de estudio. La "S" corresponde al río Santiago; la " $\mathrm{H}$ " al río Huaynamota; y la "P" a la Presa (Fuente: Ibarra-Montoya et al., 2010). 
IBARRA-MONTOYA, J. L.; RANGEL-PERAZA, G.; GONZÁLEZ-FARIAS, F. A.; ANDA, J.; MARTÍNEZMEYER, E.; MACIAS-CUELLAR, H. M. Uso del modelado de nicho ecológico como una herramienta para predecir la distribución potencial de Microcystis sp (cianobacteria) en la Presa Hidroeléctrica de Aguamilpa, Nayarit, México. Ambi-Agua, Taubaté, v. 7, n. 1, p. 218-234, 2012. (http://dx.doi.org/10.4136/ambiagua.607)

La P. H. de Aguamilpa tiene un clima cálido subhúmedo, se ubica en la categoría Awo (W), que corresponde al clima más seco de los cálidos subhúmedos (García, 1973). Según Rangel-Peraza et al. (2009) presenta un régimen hidrológico estacional, con lluvias en verano y dos épocas de estiaje, uno frío en los meses de noviembre a febrero y uno cálido de marzo a mayo. Respecto al clima de la cuenca del río Santiago, éste es variado, va desde semiseco y semicálido en las regiones más altas, hasta semicálido y subhúmedo en la parte central, y templado, semifrío y frío en las serranías. La temperatura media anual oscila entre 24 y $26^{\circ} \mathrm{C}$, con una máxima de $40{ }^{\circ} \mathrm{C}$ y mínima de $-2{ }^{\circ} \mathrm{C}$ (INEGI, 2006b). Por su parte la cuenca del río Huaynamota presenta una temperatura media anual que va de $\operatorname{los} 18^{\circ} \mathrm{C}$ a $\operatorname{los} 26^{\circ} \mathrm{C}$ (INEGI, 2006b).

\section{MATERIAL Y MÉTODOS}

\subsection{Modelo de Nicho Ecológico}

Se utilizó el enfoque de Máxima Entropía (MAXENT) para modelar el nicho ecológico y predecir la distribución de Microcystis sp. Para este trabajo se utilizó la versión de escritorio 3.3.0. de noviembre 2011 (Phillips et al., 2006). En general, este algoritmo detecta las relaciones no aleatorias entre dos conjuntos de datos: a) los registros georeferenciados de la presencia de la especie, y b) un conjunto de coberturas tipo "raster", de datos digitales que representan a las variables físico-químicas y biológicas pertinentes para determinar la distribución de la especie en una escala particular de análisis (Phillips et al., 2006).

MAXENT ha sido utilizado ampliamente para estimar la distribución potencial de los grupos terrestres con una gran precisión (Anderson et al., 2002; Peterson et al., 2002a, 2002b), y los pocos resultados que se han obtenido de las exploraciones en donde se ha usado para especies acuáticas han sido prometedores (Ibarra-Montoya et al., 2010).

Una explicación detallada de los aspectos técnicos de MAXENT se puede revisar en Phillips et al. (2006), sin embargo el procedimiento general aplicado para construir los modelos de nicho en el embalse de Aguamilpa es el siguiente:

1. Selección de las estaciones de muestreo. Se eligieron ocho estaciones de muestreo, según Rangel-Peraza et al. (2009), tres se ubican en la Presa: P6, P7 y P8; tres sobre el río Santiago: S1, S2 y S3; y dos en el curso del río Huaynamota: H4 y H5 (Figura 1). Todas las estaciones fueron georeferenciadas con un geoposicionador satelital (GPS map, 178C Sounder, marca GARMIN).

En cada una de las estaciones se registraron, a nivel superficial $(1 \mathrm{~m})$, once parámetros físicos, químicos y biológicos del agua, utilizando una sonda multiparámetro YS1 (Modelo 6600D), que fue calibrada in situ antes de cada muestreo. Los parámetros medidos fueron: temperatura, conductividad, sólidos disueltos totales, oxígeno disuelto, $\mathrm{pH}$, potencial de óxido reducción, turbidez, concentración de algas verde-azules, clorofila total, fósforo y nitrógeno (Tabla 1).

La selección de las estaciones de muestreo, tanto para los parámetros ambientales como para las colectas de fitoplancton, se basa en encontrar los lugares adecuados para generar un mapa de interpolación. Por lo tanto, las estaciones fueron seleccionadas según los siguientes criterios: i) ubicación longitudinal sobre los cauces de los ríos Santiago y Huaynamota; ii) ubicación en el área de confluencia donde se interconectan ambos afluentes de los ríos y iii) ubicación cercana a la cortina de la presa para incluir todo el embalse.

2. Colecta e identificación de la especie. Se tomaron muestras superficiales en botellas plásticas de 1 litro de capacidad, que fueron fijadas con acetato de lugol al 1 \% (Ferrario et al., 1995). Las muestras fueron analizadas con ayuda de un microscopio invertido de acuerdo al 
IBARRA-MONTOYA, J. L.; RANGEL-PERAZA, G.; GONZÁLEZ-FARIAS, F. A.; ANDA, J.; MARTÍNEZMEYER, E.; MACIAS-CUELLAR, H. M. Uso del modelado de nicho ecológico como una herramienta para predecir la distribución potencial de Microcystis sp (cianobacteria) en la Presa Hidroeléctrica de Aguamilpa, Nayarit, México. Ambi-Agua, Taubaté, v. 7, n. 1, p. 218-234, 2012. (http://dx.doi.org/10.4136/ambiagua.607)

método de Utermmohl (Hasle, 1978). En cada muestra se identificó a Microcystis sp., con ayuda de las claves taxonómicas.

3. Generación de las coberturas de los parámetros físico-químicos y biológicos. Utilizando los datos anuales registrados de cada parámetro (muestreos bimensuales), se obtuvieron los promedios por cada una de las épocas climáticas presentes en el embalse de Aguamilpa (Tabla 1); estiaje frío (diciembre 2008-febrero 2009) y estiaje cálido (abril-junio 2009). Cabe mencionar que para la época de lluvias (agosto-octubre 2008) no se registro la presencia de esta especie.

Se generaron las coberturas con los promedios de las épocas climáticas, éstas se construyeron utilizando un polígono que delimita al cuerpo de agua del embalse, el cual fue creado a una cota de 300 metros sobre el nivel del mar (msnm), a partir de las Curvas de Nivel y el Modelo Digital de Elevación (Escala 1:250,000) de CONAGUA (México, 2006). Estas coberturas, mapas tipo "raster", se generaron utilizando la técnica IDW de interpolación (Distancia Inversa Ponderada) (Delaney, 1999), en el Sistema de Información Geográfica ArcMap 9.2. ${ }^{\circledR}$ (GIS, ESRI, 1999-2001), enmascarando el análisis solo para el cuerpo de agua de la Presa. La resolución definitiva (tamaño de píxel) de todas las coberturas fue de $1 \mathrm{~m}^{2}$ (Zambrano et al., 2006).

4. Modelo de nicho ecológico. Utilizando las coberturas de los parámetros y los datos de presencia de Microcystis sp., se llevó a cabo el modelaje con MAXENT. El $75 \%$ de los registros de presencia fueron utilizados como puntos de entrenamiento y el $25 \%$ como puntos de validación. Se utilizó un umbral de convergencia de $10^{-5}$ con 1000 iteraciones como límite superior para cada corrida. Para estimar la capacidad de predicción de cada modelo, se analizó el área bajo la curva (AUC), salida gráfica donde se observa la capacidad de discriminación de una presencia (sensitividad) versus la capacidad de discriminación de una ausencia (especificidad) (Phillips y Dudik, 2008). El formato de salida analizado es el acumulativo (rango de 0 a 100), el cual indica una idoneidad de hábitat relativa de cada píxel más no una probabilidad de ocurrencia de la especie (salida logística). Se usó la prueba de Jackknife (Sokal y Rohlf, 1995; Phillips et al., 2006) para calcular la contribución relativa de cada variable al modelo, los resultados de esta evaluación se expresan con una medida conocida como "ganancia". Esta información es de suma importancia para evidenciar los requerimientos ecológicos de la especie, y que probablemente determinen el área de distribución potencial propia. Ambos análisis, el AUC y la prueba de Jackknife están implementados en MAXENT.

5. Desarrollo de los modelos de predicción. Debido a que MAXENT produce resultados de alguna manera diferentes de una corrida a la siguiente, utilizando la misma entrada de datos, se desarrollaron 100 modelos de predicción independientes para la especie, y se eligieron un subconjunto de los mejores 10 modelos basado en dos criterios: i) fue seleccionado un primer conjunto de 20 modelos con menos del $10 \%$ de error por omisión y; ii) de ellos, se seleccionaron los 10 modelos más cercanos a la media en las zonas donde la especie fue predicha como presente (Anderson et al., 2003). Estos 10 modelos fueron examinados en un Sistema de Información Geográfica (SIG), y se generó un mapa de consenso con los valores de píxel de 0 a 10, donde el 0 representa las áreas en las que todos los modelos predicen la ausencia de la especie y el 10 representa las zonas donde todos los modelos están de acuerdo en la predicción de la presencia de la especie. 
IBARRA-MONTOYA, J. L.; RANGEL-PERAZA, G.; GONZÁLEZ-FARIAS, F. A.; ANDA, J.; MARTÍNEZMEYER, E.; MACIAS-CUELLAR, H. M. Uso del modelado de nicho ecológico como una herramienta para predecir la distribución potencial de Microcystis sp (cianobacteria) en la Presa Hidroeléctrica de Aguamilpa, Nayarit, México. Ambi-Agua, Taubaté, v. 7, n. 1, p. 218-234, 2012. (http://dx.doi.org/10.4136/ambiagua.607)

\section{RESULTADOS}

En el modelo la capacidad de predicción para los datos de prueba generó un AUC de 0.903, lo que indica que la habilidad del modelo para clasificar las presencias fue bueno (Parolo et al., 2008). Así mismo, las variables que tuvieron el mayor poder de predicción cuando fueron analizadas independientemente son: en el estiaje frío, el fósforo (AUC $=0.93$ ), las algas verde-azules $(\mathrm{AUC}=0.78)$ y la turbidez $(\mathrm{AUC}=0.65)$; mientras que la variable con el menor poder de predicción fue el potencial de óxido reducción ( $\mathrm{AUC}=0.23$ ); en el estiaje cálido las variables con mayor poder de predicción fueron: el nitrógeno (AUC=0.87), el fósforo $(\mathrm{AUC}=0.74)$ y la temperatura $(\mathrm{AUC}=0.73)$; mientras que la variable con menor poder de predicción fue también el potencial de óxido reducción (AUC=0.46) (Tabla 2).

Tabla 1. Valores promedio de los parámetros físicos, químicos y biológicos que se usaron para generar las coberturas del cuerpo de agua de los diferentes sitios de muestreo, durante el periodo de agosto 2008 a junio 2009. Loc=localidad; T=temperatura del agua; Cond=conductividad; $\mathrm{SDT}=$ sólidos disueltos totales; $\mathrm{OD}=$ oxígeno disuelto; $\mathrm{ORP}=$ potencial de óxido reducción; Tur=turbidez; F=fósforo; N=nitrógeno.

\begin{tabular}{|c|c|c|c|c|c|c|c|c|c|c|c|}
\hline \multicolumn{12}{|c|}{ Estiaje Frío } \\
\hline Loc & $\begin{array}{c}\mathrm{T} \\
\left({ }^{\circ} \mathrm{C}\right)\end{array}$ & $\begin{array}{c}\text { Cond } \\
\left(\mu \mathrm{S} \mathrm{cm} \mathrm{cm}^{-1}\right)\end{array}$ & $\begin{array}{l}\text { SDT } \\
\left(\mathrm{g} \mathrm{l}^{-1}\right)\end{array}$ & $\begin{array}{c}\text { OD } \\
\left(\mathrm{mg} \mathrm{l}^{-1}\right)\end{array}$ & pH & ORP & $\begin{array}{c}\text { Tur } \\
\text { (NTU) }\end{array}$ & $\begin{array}{c}\text { Algas } \\
\left(\text { cel ml }^{-1}\right)\end{array}$ & $\begin{array}{c}\text { Clorofila } \\
\left(\mu \mathrm{g} \mathrm{l}^{-1}\right)\end{array}$ & $\begin{array}{c}F \\
\left(\mathrm{mg} \mathrm{l}^{-1}\right)\end{array}$ & $\begin{array}{c}\mathrm{N} \\
\left(\mathrm{mgl}^{-1}\right)\end{array}$ \\
\hline $\mathrm{S} 1$ & 25.06 & 0.259 & 0.168 & 3.92 & 7.6 & 52.43 & 52.72 & 525 & 5.36 & 2.22 & 0.24 \\
\hline $\mathrm{S} 2$ & 24.94 & 0.254 & 0.165 & 4.02 & 7.7 & 45.97 & 54.00 & 488 & 5.19 & 2.21 & 0.26 \\
\hline $\mathrm{S} 3$ & 25.00 & 0.257 & 0.167 & 4.00 & 7.6 & 40.55 & 54.45 & 558 & 5.23 & 2.24 & 0.20 \\
\hline $\mathrm{H} 4$ & 24.52 & 0.173 & 0.112 & 2.65 & 7.3 & 56.11 & 42.61 & 842 & 3.83 & 0.71 & 0.13 \\
\hline H5 & 24.96 & 0.168 & 0.110 & 3.20 & 7.3 & 51.08 & 51.22 & 398 & 4.54 & 0.69 & 0.16 \\
\hline P6 & 24.92 & 0.230 & 0.149 & 5.29 & 7.6 & 46.34 & 55.69 & 373 & 5.03 & 2.39 & 0.27 \\
\hline P7 & 24.73 & 0.231 & 0.150 & 6.31 & 7.6 & 55.51 & 46.20 & 757 & 5.39 & 2.43 & 0.28 \\
\hline P8 & 24.72 & 0.229 & 0.149 & 6.12 & 7.6 & 48.26 & 49.66 & 352 & 4.41 & 2.37 & 0.25 \\
\hline
\end{tabular}

\begin{tabular}{|c|c|c|c|c|c|c|c|c|c|c|c|}
\hline \multicolumn{12}{|c|}{ Estiaje Cálido } \\
\hline Loc & $\begin{array}{c}\mathbf{T} \\
\left({ }^{\circ} \mathbf{C}\right)\end{array}$ & $\underset{\left(\mu S \text { cm }^{-1}\right)}{\text { Cond }}$ & $\begin{array}{l}\text { SDT } \\
\left(\mathrm{g} \mathrm{l}^{-1}\right)\end{array}$ & $\underset{\left(\mathrm{mg} \mathrm{l}^{-1}\right)}{\text { OD }}$ & pH & ORP & $\begin{array}{c}\text { Tur } \\
\text { (NTU) }\end{array}$ & $\underset{\left(\text { cel } \mathbf{m l}^{-1}\right)}{\text { Algas }}$ & $\begin{array}{c}\text { Clorofila } \\
\left(\mu \mathrm{g} \mathrm{l^{-1 }}\right)\end{array}$ & $\underset{\left(\mathrm{mg} \mathrm{l}^{-1}\right)}{\mathbf{F}}$ & $\underset{\left(\mathbf{m g l}^{-\mathbf{1}}\right)}{\mathbf{N}}$ \\
\hline $\mathrm{S} 1$ & 27.89 & 0.289 & 0.188 & 7.32 & 8.6 & 91.50 & 27.63 & 1605 & 6.7 & 0.77 & 0.78 \\
\hline $\mathrm{S} 2$ & 28.81 & 0.280 & 0.182 & 8.06 & 8.7 & 87.65 & 26.79 & 1376 & 6.4 & 0.75 & 0.72 \\
\hline S3 & 28.96 & 0.278 & 0.181 & 8.46 & 8.6 & 88.65 & 27.80 & 1566 & 7.3 & 0.74 & 0.74 \\
\hline $\mathrm{H} 4$ & 27.47 & 0.288 & 0.187 & 3.73 & 7.5 & 120.82 & 19.46 & 1092 & 5.5 & 0.28 & 1.02 \\
\hline H5 & 27.90 & 0.261 & 0.169 & 7.41 & 8.0 & 54.01 & 21.28 & 1170 & 3.3 & 0.26 & 1.05 \\
\hline P6 & 27.74 & 0.261 & 0.167 & 8.58 & 8.3 & 108.49 & 28.28 & 968 & 6.2 & 0.73 & 0.65 \\
\hline P7 & 27.03 & 0.256 & 0.166 & 9.23 & 8.2 & 90.85 & 33.71 & 7416 & 6.7 & 0.71 & 0.63 \\
\hline P8 & 26.32 & 0.264 & 0.172 & 8.15 & 8.2 & 126.02 & 29.29 & 1507 & 7.5 & 0.73 & 0.64 \\
\hline
\end{tabular}

Las predicciones del modelo para Microcystis sp., durante el estiaje frío, muestran que tiene una distribución en todo el sistema de Aguamilpa (Figura 2), aunque el modelo predice una mayor probabilidad de encontrarse en el afluente del río Huaynamota y en la cortina de la Presa. Durante el estiaje cálido esta especie tiene una mayor probabilidad de encontrase en la cuenca alta del río Huaynamota y en la Confluencia (P6), así mismo, aunque con menor probabilidad en la cuenca alta del río Santiago.

Las zonas que el modelo predijo como más probables para la distribución de Microcystis $s p$., están asociadas principalmente con la presencia de nutrientes como el fósforo y nitrógeno (Tabla 2). 
Tabla 2. Valores de AUC, para las variables analizadas, en las distintas épocas del año, según el modelo de nicho ecológico. $\mathrm{T}=$ =temperatura del agua; Cond=conductividad; SDT=sólidos disueltos totales; $\mathrm{OD}=$ =xígeno disuelto; ORP=potencial de óxido reducción; Tur=turbidez; F=Fósforo; N=Nitrógeno.

\begin{tabular}{c|cc}
\hline Variable & Estiaje Frío & Estiaje Cálido \\
\hline T & 0.64 & $\mathbf{0 . 7 3}$ \\
Cond & 0.56 & 0.36 \\
SDT & 0.60 & 0.65 \\
OD & 0.54 & 0.57 \\
pH & 0.63 & 0.69 \\
ORP & 0.23 & 0.46 \\
Tur & $\mathbf{0 . 6 5}$ & 0.72 \\
Algas & $\mathbf{0 . 7 8}$ & 0.64 \\
Clorofila & 0.67 & 0.68 \\
F & $\mathbf{0 . 9 3}$ & $\mathbf{0 . 7 4}$ \\
N & 0.61 & $\mathbf{0 . 8 7}$ \\
\hline
\end{tabular}

Por ejemplo, durante el estiaje frío, todo el sistema Aguamilpa tiene probabilidad de ser un nicho idóneo para la distribución de Mycrocystis sp. Es muy probable que este fenómeno se deba al aumento de flujo de agua que se da en la época de lluvias y que se mantiene hasta el estiaje frío, dicho aumento estará dado por los escurrimientos, particularmente desde las cuencas altas de los ríos Santiago y Huyanamota que traen consigo diferentes nutrientes, principalmente fósforo y nitrógeno al cuerpo de agua del sistema. Lo anterior, ocasiona que la mayor parte del cuerpo de agua del embalse mantenga condiciones ambientales adecuadas para el crecimiento y desarrollo de esta especie.

Durante el estiaje cálido, se observan dos zonas de mayor probabilidad de distribución de Microcystis sp: i) en la cuenca alta del río Huaynamota; y ii) en la confluencia de ambos ríos. Este fenómeno es probable que se deba a que estas áreas mantienen altas concentraciones de nitrógeno y fósforo, como una consecuencia del poco movimiento que presenta el agua en esta época. Lo anterior genera un ambiente ideal para el crecimiento y abundancia de esta especie.

Es posible que dichos nutrientes sean producto de las actividades antropogénicas, agrícolas, industriales y urbanas, principalmente por la cuenca del río Santiago y las actividades de tala, y la creciente deforestación en la cuenca alta del río Huaynamota, lo cual induce erosión de suelos, transporte de nutrientes y descomposición de los ciclos biogeoquímicos naturales de la cuenca, durante la época de lluvias, a los arroyos y ríos que forman la red hidrográfica del sistema Aguamilpa. 
IBARRA-MONTOYA, J. L.; RANGEL-PERAZA, G.; GONZÁLEZ-FARIAS, F. A.; ANDA, J.; MARTÍNEZMEYER, E.; MACIAS-CUELLAR, H. M. Uso del modelado de nicho ecológico como una herramienta para predecir la distribución potencial de Microcystis sp (cianobacteria) en la Presa Hidroeléctrica de Aguamilpa, Nayarit, México. Ambi-Agua, Taubaté, v. 7, n. 1, p. 218-234, 2012. (http://dx.doi.org/10.4136/ambiagua.607)

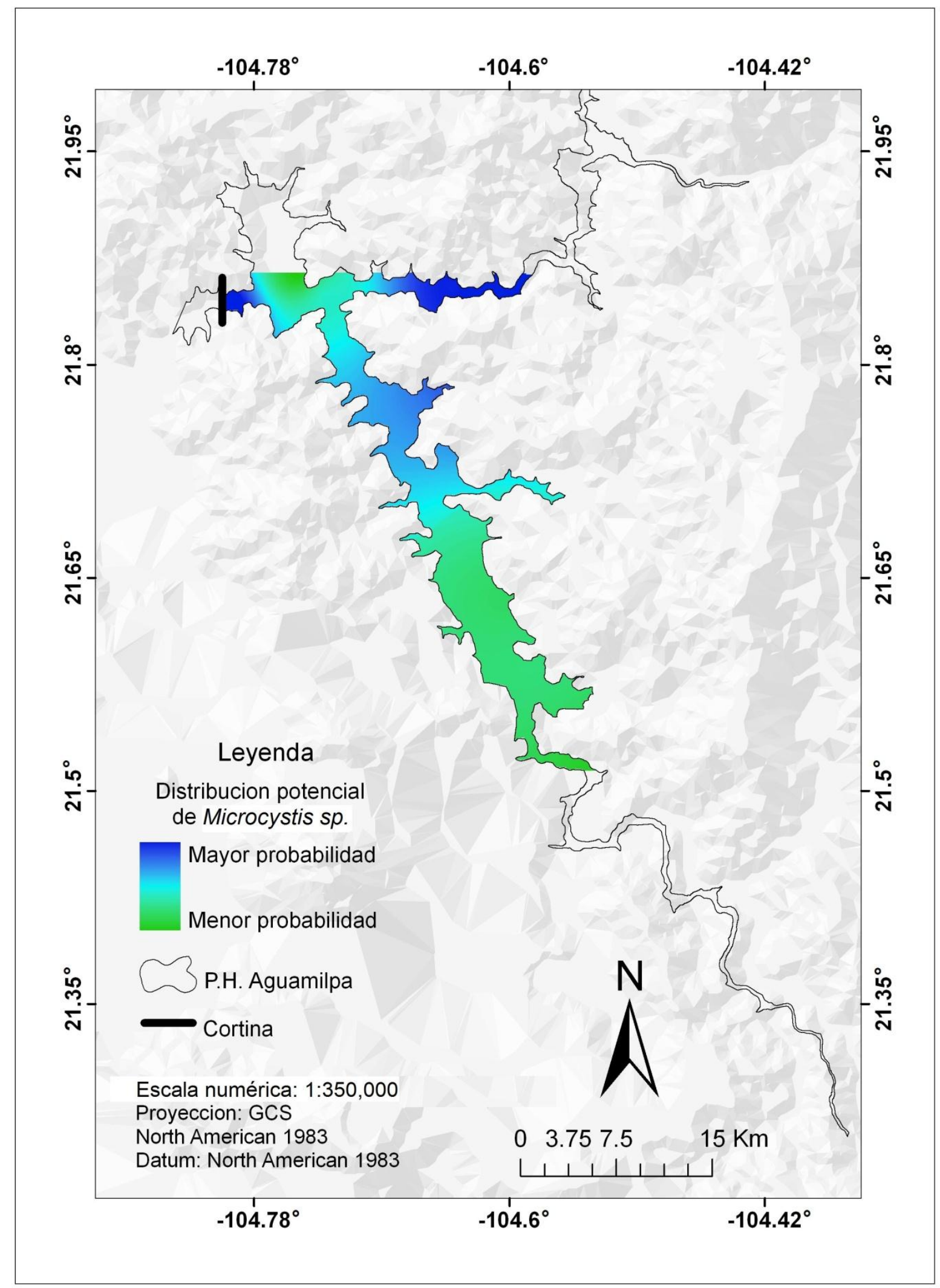

Figura 2. Zonas potenciales de distribución de Microcystis sp., en el embalse de Aguamilpa, durante el estiaje frío. Las probabilidades fueron las predichas por el modelo de nicho ecológico. 
IBARRA-MONTOYA, J. L.; RANGEL-PERAZA, G.; GONZÁLEZ-FARIAS, F. A.; ANDA, J.; MARTÍNEZMEYER, E.; MACIAS-CUELLAR, H. M. Uso del modelado de nicho ecológico como una herramienta para predecir la distribución potencial de Microcystis sp (cianobacteria) en la Presa Hidroeléctrica de Aguamilpa, Nayarit, México. Ambi-Agua, Taubaté, v. 7, n. 1, p. 218-234, 2012. (http://dx.doi.org/10.4136/ambiagua.607)

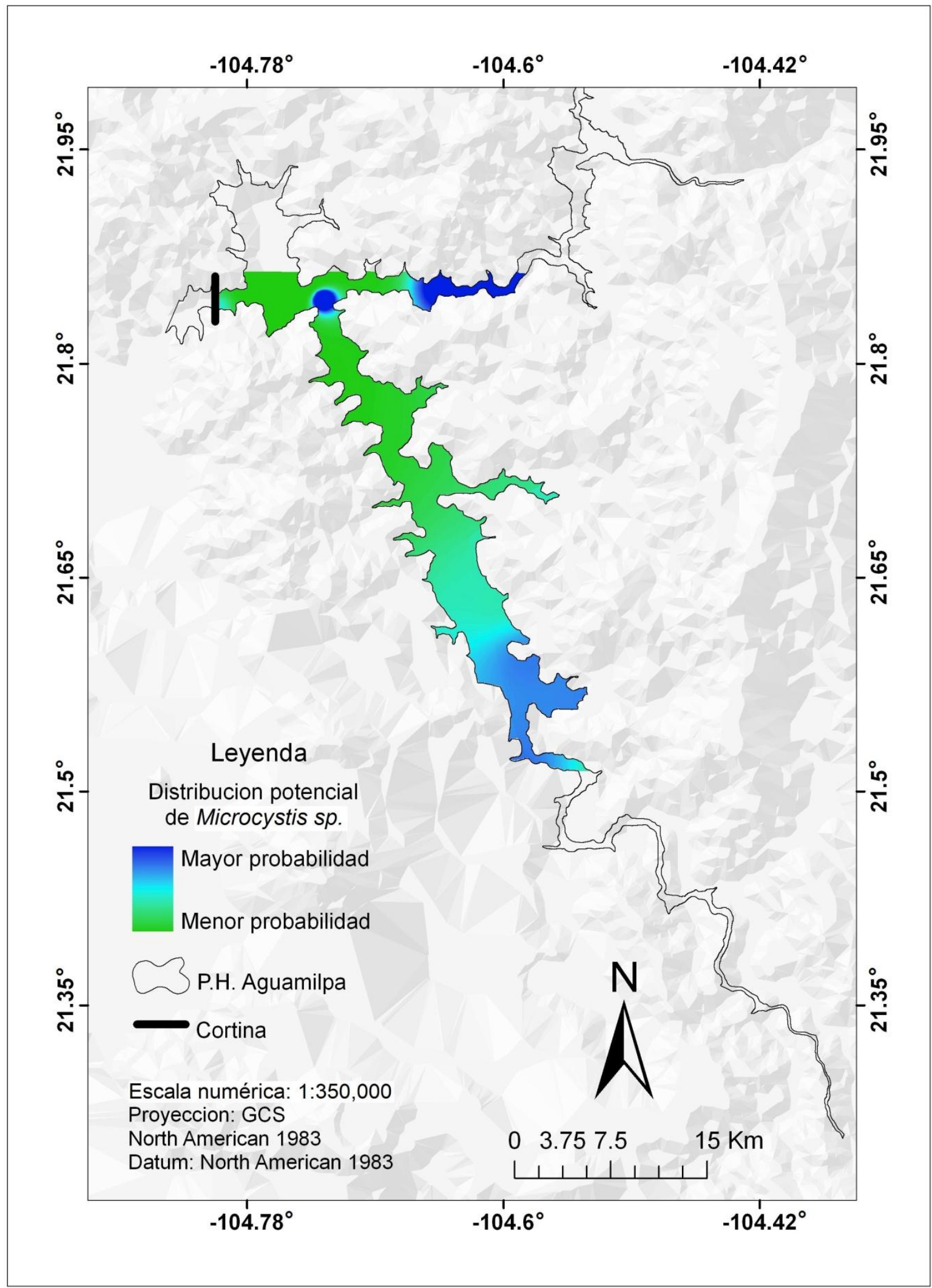

Figura 3. Zonas potenciales de distribución de Microcystis sp., en el embalse de Aguamilpa, durante el estiaje cálido. Las probabilidades fueron las predichas por el modelo de nicho ecológico. 

predecir la distribución potencial de Microcystis sp (cianobacteria) en la Presa Hidroeléctrica de Aguamilpa, Nayarit, México. Ambi-Agua, Taubaté, v. 7, n. 1, p. 218-234, 2012. (http://dx.doi.org/10.4136/ambiagua.607)

\section{DISCUSIÓN}

El modelado de nicho ecológico (junto con otras herramientas de análisis espacial, sobre todo los SIG, Sistemas de Información Geográfica) es un enfoque útil para futuros estudios en los sistemas terrestres (Chen y Peterson, 2002; Anderson y Martínez-Meyer, 2004; Chefauri et al., 2005). Sin embargo, este enfoque ha sido poco utilizado en los sistemas acuáticos (Wiley et al., 2003; McNyset, 2005; Ibarra-Montoya et al., 2010), en gran parte, debido a la falta de información ambiental (Iguchi et al., 2004). A pesar de esta limitación, la predicción de ocurrencia de la especie con base en las localidades incluidas para la construcción de este modelo, fue bastante bueno. El análisis de la AUC obtuvo una predicción el $90 \%$, lo que sugiere que MAXENT generó un buen desempeño en la predicción de la distribución potencial de Microcystis sp.

En otros estudios donde se utilizan modelos de nicho ecológico es frecuente que se presenten sobreestimaciones, éstas pueden tener varias causas, algunas son algorítmicas y otras son conceptuales y metodológicas: (1) Estudios recientes de la comparación de modelos con MAXENT y GARP (Genetic Algorithm for Rule-Set Prediction) muestran una frecuente sobreestimación (clasificar erróneamente las ausencias) con respecto a otros métodos (Elith, 2006; Pearson et al., 2006). Sin embargo, estos análisis se centraron en la capacidad de los algoritmos para interpolar (en vez de extrapolar) analizando los modelos construidos con puntos de presencia-ausencia seleccionados al azar de un conjunto de datos más grande. En los estudios diseñados explícitamente para poner a prueba la capacidad de extrapolación (predicción de las presencias en las zonas donde no hay datos de muestreo) MAXENT ha funcionado bien (Peterson, 2001). (2) La sobreestimación también se debe a la naturaleza del enfoque en relación con la historia natural del grupo. El enfoque del modelado de nicho ecológico se centra en las condiciones ambientales donde una especie puede persistir (es decir, su nicho ecológico) y no incluye los factores históricos y ecológicos que provocan que las especies ocupen en su totalidad la extensión espacial de sus nichos ecológicos (por ejemplo, las barreras biogeográficas y las interacciones) (Soberón y Peterson, 2005). Por lo tanto, la ausencia de una especie de un lugar determinado no significa necesariamente la ausencia de su nicho ecológico. Esto es particularmente importante para Microcystis sp., porque el elevado número de individuos en general, indican una historia muy dinámica de aislamiento y su posterior abundancia bajo condiciones físico-químicas, biológicas y de nutrientes del agua. (3) Por último, la falta de información ambiental de cuerpos de agua representa una importante limitación metodológica que pudiera influir en los resultados.

Claramente, la producción y la incorporación de la información ambiental específicamente en relación con los sistemas de agua (por ejemplo, profundidad, $\mathrm{pH}$, nutrientes y contenido de oxígeno) mejoran en gran medida la calidad de los modelos.

Dentro del grupo de cianobacterias la especie Microcystis sp., es de las más distintivas en los cuerpo de agua dulce. Varias poblaciones de esta especie poseen rasgos biológicos y ecológicos únicos, como la producción de sustancias alelopáticas, para eliminar la competencia de otros organismos del medio, y para evitar ser depredadas por otros organismos (Shapiro, 1973).

Al igual que los demás miembros de las cianobacterias, requieren de la energía luminosa para realizar la fotosíntesis, sin embargo, Microcystis sp., tiene la capacidad de sintetizar diversos pigmentos que le permiten capturar la luz de manera muy eficiente, absorbiendo la radiación electromagnética en longitudes de onda de 500 hasta $600 \mathrm{~nm}$, que difícilmente son empleadas por otros organismos del fitoplancton, incluso pueden vivir en ambientes con solo luz verde (Mur et al., 1977). 

predecir la distribución potencial de Microcystis sp (cianobacteria) en la Presa Hidroeléctrica de Aguamilpa, Nayarit, México. Ambi-Agua, Taubaté, v. 7, n. 1, p. 218-234, 2012. (http://dx.doi.org/10.4136/ambiagua.607)

Microcystis sp., presenta vesículas de gas, las cuales le permiten migrar y posicionarse verticalmente en la columna de agua (Walsby, 1997). De acuerdo a Reynolds (1999), la tasa de crecimiento de las cianobacterias, particularmente de Microcystis sp., es mucho menor que la de microalgas, debido a esto, se requiere de tiempos de retención del agua prolongados para permitir así las floraciones algales. Se pensaba que esta especie al igual que otras cianobacterias dependían del nitrógeno y fósforo en altas concentraciones, sin embargo, se ha visto que pueden crecer incluso cuando estos dos elementos están en condiciones limitantes; ya que esta especie tienen la capacidad de almacenar fósforo, lo cual le permite hasta cuatro divisiones celulares, lo que correspondería a un incremento sustancial en biomasa (Downing et al., 2005). Presentan una estabilidad poblacional, que les permite prevalecer por más tiempo una vez establecidas (Chorus y Bartram, 1999).

La temperatura óptima para el crecimiento es mayor que la necesaria $\left(15-30{ }^{\circ} \mathrm{C}\right)$, lo cual explicaría porque en cuerpos de agua templados y boreales se dan los florecimientos durante el verano, mientras que en un sistema con clima tropical como el caso de Aguamilpa, están presentes en ambos estiajes, el frío y el cálido. Por otro lado, Microcystis sp., forma scums, (colonias) de células cocoides o filamentos, lo que le proporciona la capacidad de flotar y posicionarse en la parte más alta de la columna de agua, hasta hallar la intensidad luminosa favorable para su crecimiento. Esto significa que la presencia de esta especie no esta relacionada solo al nivel de eutrofización del medio, por lo que puede encontrarse en aguas mesotróficas, eutróficas e hipertróficas, pero la cantidad de biomasa estará relacionada con la cantidad de nutrientes disponibles (Mur et al., 1999).

Además de sus diferentes estrategias ecofisiológicas, su diversidad poblacional y dominancia en el sistema Aguamilpa, tiene un papel de importancia ecológica para las demás especies del fitoplancton. Sin embargo, y a pesar de su importancia se ha hecho poco para evaluar su presencia en el sistema, y la amenaza de que esta especie provoque "blooms" y daños a la biota es probable.

\subsection{Presencia de Microcystis sp., en la P. H. Aguamilpa}

En el modelo se identificaron zonas muy extensas en donde es probable que se distribuya Microcytis sp. Tanto en la cuenca del río Huaynamota como en la del río Santiago, se predice una distribución continua durante el estiaje frío, mientras que durante el estiaje cálido la zona de distribución potencial se reduce a tres áreas dentro del sistema Aguamilpa. Estos resultados pueden deberse a dos factores: (1) una sobreestimación del modelo, y (2) a que en estos ambos estiajes, el nicho ecológico de Microcystis sp., es todo el sistema Aguamilpa.

Ambas situaciones son posibles. Como ya se menciono anteriormente, la sobreestimación (es decir, error de omisión) es común en modelos de nicho ecológico. Por otra parte, y aunque se hizo el esfuerzo por contar con los estudios de campo (colectas en más estaciones), todavía siguen siendo incompletos, por lo que varias estaciones más aún pueden producir nuevos registros de esta especie. Este fenómeno podría ser la respuesta al hecho de que en la época de lluvias no se encontraran registros de esta especie.

\subsection{Uso de suelo}

Microcystis sp., es sensible a los cambios en la calidad de agua (Chorus, 2001). Por lo tanto, para mantener sus poblaciones bajo un esquema "normal" (es decir, sin producir toxinas), es importante tener en cuenta la calidad de agua. Sin embargo, las zonas con mayor probabilidad de presencia de la especie, como el río Santiago y la cuenca alta del río Huaynamota, han sufrido durante el siglo pasado, importantes transformaciones de la cubierta vegetal, utilizando el suelo para terrenos agrícolas, para las actividades de pastoreo, urbanas e industriales (Mercado-Silva et al., 2002). Estos usos de la tierra modifican directamente la 
IBARRA-MONTOYA, J. L.; RANGEL-PERAZA, G.; GONZÁLEZ-FARIAS, F. A.; ANDA, J.; MARTÍNEZMEYER, E.; MACIAS-CUELLAR, H. M. Uso del modelado de nicho ecológico como una herramienta para predecir la distribución potencial de Microcystis sp (cianobacteria) en la Presa Hidroeléctrica de Aguamilpa, Nayarit, México. Ambi-Agua, Taubaté, v. 7, n. 1, p. 218-234, 2012. (http://dx.doi.org/10.4136/ambiagua.607)

calidad de agua e incrementan los nutrientes (principalmente fósforo y nitrógeno) a través de la contaminación, la eutrofización (por la entrada de fertilizantes), y la erosión del suelo.

De a cuerdo con los análisis, la mayoría de las áreas en donde habita Microcystis sp., son zonas continuas dentro del cuerpo de agua (Figuras 2 y 3) y las áreas que el modelo predice con una mayor probabilidad de presencia de la especie han sido transformadas drásticamente por la actividad humana. En esta áreas Microcystis sp., sobrevive principalmente, donde las tasas de renovación de agua son altas y la contaminación y otros factores de perturbación se amortiguan (García-Cabrera, 2006). El uso de suelo, en torno a la transformación de los cuerpo de agua con certeza ha afectado directa o indirectamente a la biota acuática, probablemente incluso causando que otras especies de fitoplancton sean eliminadas del cuerpo de agua en Aguamilpa.

Por ultimo, con estos análisis se argumenta que el elemento limitante para el crecimiento de las cianobacterias, en especial para Microcystis sp., en ambientes tropicales, es además del nitrógeno, el fósforo (Henry et al., 1985; Dávalos et al., 1989). Con esto, se tiene información de que en los cuerpos de agua dulce ubicados en zonas tropicales son ambos nutrientes los que en gran medida determinan la distribución de las cianobacterias, y no solo el nitrógeno como se pensaba (Philips et al., 1993).

\section{CONCLUSIÓN}

El modelo de nicho ecológico demostró ser una metodología confiable para la predicción de la distribución potencial de Microcystis sp., en el cuerpo de agua de la P. H. Aguamilpa. El estudio se caracterizó por presentar áreas aisladas de mayor probabilidad de presencia de esta especie durante el estiaje cálido, mientras que en el estiaje frió, Microcystis sp., se puede encontrar en casi todo el sistema Aguamilpa. Se sugiere que la presencia de esta especie se encuentra asociada al cambio en el uso de suelo y vegetación y a los procesos tróficos derivados de las actividades antropogénicas que se llevan a cabo en la cuenca del sistema. Por ejemplo, en la cuenca alta del rio Huaynamota principalmente la agricultura y la ganadería, y por el río Santiago las actividades industriales.

Por otro lado, el estudio documenta que existe una dinámica entre los nutrientes (fósforo y nitrógeno) que en gran medida determina la distribución de Microcystis $s p$.

Esta metodología se recomienda para ser utilizada tanto en este sistema como en otros, para ayudar a identificar las zonas potenciales de distribución de Microcystis sp, y con esto localizar a lo largo de la cuenca las áreas geográficas donde se recomiende realizar acciones de restauración y conservación, principalmente del uso de suelo y vegetación, y que tales esfuerzos puedan orientarse en una estrecha coordinación entre las poblaciones locales, autoridades y la academia.

\section{AGRADECIMIENTOS}

Al Dr. José de Anda, del Centro de Investigación y Asistencia en Tecnología del Estado de Jalisco, y al Dr. Fernando González-Farias, del Instituto de Ciencias del Mar y Limnología, Universidad Nacional Autónoma de México, por su asesoría y orientación, A todos los colaboradores de este trabajo, especialmente a la Biol. Karla Gutiérrez, por su auxilio y ayuda en el desarrollo y aplicación de SIG. Al proyecto Aguamilpa, clave: CB-CONACYT-200658119 por la beca otorgada y al Consejo Mexiquense de Ciencia y Tecnología (COMECYT), del Estado de México, por la beca otorgada. 
IBARRA-MONTOYA, J. L.; RANGEL-PERAZA, G.; GONZÁLEZ-FARIAS, F. A.; ANDA, J.; MARTÍNEZMEYER, E.; MACIAS-CUELLAR, H. M. Uso del modelado de nicho ecológico como una herramienta para predecir la distribución potencial de Microcystis sp (cianobacteria) en la Presa Hidroeléctrica de Aguamilpa, Nayarit, México. Ambi-Agua, Taubaté, v. 7, n. 1, p. 218-234, 2012. (http://dx.doi.org/10.4136/ambiagua.607)

\section{REFERENCIAS}

ANDERSON, R. P.; LEW, D.; PETERSON, A. Evaluating predictive models of species' distributions: criteria for selecting optimal models. Ecological Modelling, v. 162, n. 3, p. 211-32, 2003. http://dx.doi.org/10.1016/S0304-3800(02)00349-6

ANDERSON, R. P.; PETERSON, A. T.; GÓMEZ-LAVERDE, M. Using niche-based GIS modelinh to test geographic predictions of competitive exclusion and competitive release in South American pocket mice. Oikos, v. 98, n. 1, p. 3-16, 2002. http://dx.doi.org/10.1034/j.1600-0706.2002.t01-1-980116.x

ANDERSON, R. P.; MARTÍNEZ-MEYER, E. Modeling species' geographic distributions for preliminary conservation assessments: an implementation with the spiny pocket mice (Heteromys) of Ecuador. Biological Conservation, v. 116, p. 167-76, 2004. http://dx.doi.org/10.1016/S0006-3207(03)00187-3

BERNAL-BROOKS, F. W.; DÁVALOS-LIND, L.; LIND, O. T. Seasonal and spatial variation in algal growth potential and growth-limiting nutrients in a shallow endorheic lake: Lake Pátzcuaro (México). Lakes \& Reservoirs: Research and Management, v. 8, n. 2, p. 83-93, 2003. http://dx.doi.org/10.1046/j.1320-5331.2003.00217.x

COOD, G. A.; BELL, S. G.; KAYA, K.; WARD, C.; BEATTIE, K.; METCALF, S. J. Cyanobacterial toxins, exposure routes and human health. European Journal of Phycology, v. 34, n. 4, p. 405-15, 1999. http://dx.doi.org/10.1080/09670269910001736462

CHEFAURI, R. M.; HORTAL, J.; LOBO, M. Potential distribution modeling, niche characterization and conservation status assessment using GIS tools: a case study of Iberian Copris species. Biological Conservation, v. 122, p. 327-38, 2005. DOI:10.1016/j.biocon.2004.08.005 http://dx.doi.org/10.1016/j.biocon.2004.08.005

CHEN, G.; PETERSON, A. T. Prioritization of areas in China for the conservation of endangered birds using modelled geographical distributions. Bird Conservation International, v. 12, p. 197-209, 2002. DOI: 10.1017/S0959270902002125 http://dx.doi.org/10.1017/S0959270902002125

CHORUS, I. Cyanotoxins - occurrence, causes, consequences. New York: Springer, 2001. $357 \mathrm{p}$.

CHORUS, I.; BARTRAM, J. (Ed.) Toxic cyanobacteria in water: a guide to their public health consequences, monitoring and management. London: Spon, 1999. p. 1-14.

DÁVALOS, L.; LIND, O. T.; DOYLE, R. D. Evaluation of phytoplankton limiting factors in Lake Chapala, Mexico: turbidity and spatial and temporal variations in algal assay response. Lake Reservoir Management, v. 5, n. 2, p. 99-104, 1989. http://dx.doi.org/10.1080/07438148909354404

DELANEY, J. Geographical information systems an introduction. Oxford: University Press, 1999. 194 p. ISBN 019550789-4.

DOWNING, T. G.; MEYER, C.; GEHRINGER, M. M.; VAN DE VENTER, M. Microcystin content of Microcystis aeruginosa is modulated by nitrogen uptake rate relative to specific growth rate or carbon fixation rate. Environmental Toxicology, v. 20, n. 3, p. 257-62, 2005. http://dx.doi.org/10.1002/tox.20106 
IBARRA-MONTOYA, J. L.; RANGEL-PERAZA, G.; GONZÁLEZ-FARIAS, F. A.; ANDA, J.; MARTÍNEZMEYER, E.; MACIAS-CUELLAR, H. M. Uso del modelado de nicho ecológico como una herramienta para predecir la distribución potencial de Microcystis sp (cianobacteria) en la Presa Hidroeléctrica de Aguamilpa, Nayarit, México. Ambi-Agua, Taubaté, v. 7, n. 1, p. 218-234, 2012. (http://dx.doi.org/10.4136/ambiagua.607)

ELITH, J. Novel methods improve prediction of species' distributions from occurrence data. Ecography, v. 29, n. 2, p. 129-51, 2006. http://dx.doi.org/10.1111/j.2006.0906-7590.04596.x

FERRARIO, M. E.; SAR, E.; SALA, S. E. Metodología básica para el estudio del fitoplancton con especial referencia a las diatomras. In: ALVEAL, K.; FERRARIO, M. E.; OLVEIRA, E. C.; SAR, E. (Ed.). Manual de métodos ficológicos. Concepción: Editorial Aníbal Pinto, 1995. p. 1-24.

FERRIER, S. Mapping spatial pattern in biodiversity for regional conservation planning: where to from here? Systematic Biology, v. 51, n. 2, p. 331-63, 2002. http://dx.doi.org/10.1080/10635150252899806

GARCÍA-CABRERA, J. Plancton como indicador de calidad del agua en la presa Aguamilpa. Ingeniería Hidráulica en México, v. 22, n. 1, p. 103-16, 2006.

GARCÍA, E. Modificaciones al sistema de clasificación climática de Köppen (para adaptarlo a las condiciones de la República Mexicana). Ciudad de México: Universidad Nacional Autónoma de México, Instituto de Geografía, 1973. 246 p. ISBN 75553596.

GUISAN, A.; THUILLER, W. Predicting species distribution: offering more than simple habitat models. Ecology Letters, v. 8, n. 9, p. 993-1009, 2005.

http://dx.doi.org/10.1111/j.1461-0248.2005.00792.x

HASLE, G. R. The inverted-microscope method. In: SOURNIA, A. (Ed.). Phytoplankton manual. Paris: UNESCO, 1978. p. 191-96.

HENRY, R.; HINO, K.; TUNDISI, J. G.; RIBEIRO, J. S. B. Responses of phytoplankton in lake Jacaretinga to enrichment with nitrogen and phosphorus in concentrations similar to tose of the River Solimoes (Amazon, Brazil). Archiv für Hydrobiologie, v. 103, p. 453-77, 1985.

IBARRA-MONTOYA，J. L.; RANGEL-PERAZA， G.; GONZÁLEZ-FARIAS， F.; DE ANDA, J.; ZAMUDIO-RESENDIZ, M. E.; MARTÍNEZ-MEYER, E. et al. Ecological niche model to predict the potential distribution of phytoplankton in the Aguamilpa Dam, Nayarit. México. Ambiente \& Agua - An Interdisciplinary Journal of Applied Science, v. 5, n. 3, p. 60-75, 2010. http://dx.doi.org/10.4136/ambi-agua.154

IGUCHI, K.; MATSUURA, K.; MCNYSET, K. M.; PETERSON, A. T.; SCACHETTIPEREIRA, R.; POWERS, K. A. et al. Predicting invasions of North American basses in Japan using native range data and a genetic algorithm. Transactions of the American Fisheries Society, v. 133, n. 4, p. 845-54, 2004.

http://dx.doi.org/10.1577/T03-172.1

INSTITUTO NACIONAL DE ESTADISTICA Y GEOGRAFIA - INEGI. Continuo nacional del conjunto de datos geográficos de la carta topográfica, 1:250 000, serie II. Aguascalientes, 2006a.

INSTITUTO NACIONAL DE ESTADISTICA Y GEOGRAFIA - INEGI. Conjunto nacional del conjunto de datos geográficos de la carta de climas, 1:1 000 000, serie I. Aguascalientes, 2006b. 
IBARRA-MONTOYA, J. L.; RANGEL-PERAZA, G.; GONZÁLEZ-FARIAS, F. A.; ANDA, J.; MARTÍNEZMEYER, E.; MACIAS-CUELLAR, H. M. Uso del modelado de nicho ecológico como una herramienta para predecir la distribución potencial de Microcystis sp (cianobacteria) en la Presa Hidroeléctrica de Aguamilpa, Nayarit, México. Ambi-Agua, Taubaté, v. 7, n. 1, p. 218-234, 2012. (http://dx.doi.org/10.4136/ambiagua.607)

LAMBERT, W.; SOMMER, U. Limnoecology: the ecology of lakes and streams. Oxford: University Press, 1997. 382 p. ISBN 01888897.

MAXENT. Disponible en: 〈http://www.cs.princeton.edu/ schapire/maxent>. Acceso en: nov. 2011.

MERCADO-SILVA, N.; LYONS, J. D.; SALGADO MALDONADO, G.; MEDINA NAVA, M. Validation of a fish-based index of biotic integrity or streams and rivers of central Mexico. Reviews in Fish Biology and Fisheries, v. 12, n. 2/3, p. 179-91, 2002. http://dx.doi.org/10.1023/A:1025099711746

MÉXICO. Comision Nacional Del Agua - CONAGUA. Modelo digital de elevación, escala 1:250,000. Ciudad de México: Departamento de Sistemas de Información Geográfica del Agua, 2006.

MCNYSET, K. M. Use of ecological niche modeling to predict distributions of freswater fish species in Kansas. Ecology of Freshwater Fish, v. 14, n. 3, p. 243-55, 2005. http://dx.doi.org/10.1111/j.1600-0633.2005.00101.x

MUR, L. R.; GONS, H. J.; VAN LIERE, L. Some experiments on the competition of the green algae and blue-green bacteria in light-limited environments. FEMS Microbiology Letters, v. 1, n. 6, p. 335-38, 1977.

http://dx.doi.org/10.1111/j.1574-6968.1977.tb00646.x

MUR, L. R.; SKULBERG, O. M.; UTKILEN, H. Cyanobacteria in the environment. In: CHORUS, I.; BARTRAM, J. (Ed.). Toxic cyanobacteria in water: a guide to their public health consequences, monitoring and management. London: Spon, 1999. p. 1440. ISBN 1402030223.

PAROLO, G.; ROSSI, G.; FERRARINI, A. Toward improved species niche modelling: Arnica montana in the Alps as a case study. Journal of Applied Ecology, v. 45, n. 5, p. 1410-18, 2008. http://dx.doi.org/10.1111/j.1365-2664.2008.01516.x

PEARCE, J.; LINDENMAYER, D. Bioclimatic analysis to enhance reintroduction biology of the endangered helmeted honeyeater (Lichenostomus melanops cassidix) in Southeastern Australia. Restoration Ecology, v. 6, n. 3, p. 238-43, 1998. http://dx.doi.org/10.1046/j.1526-100X.1998.00636.x

PEARSON, R. G.; THUILLER,W.; ARAÚJO, M. B.; MARTINEZ-MEYER, E.; BROTONS, L.; McCLEAN, C. et al. Model-based uncertainty in species range prediction. Journal of Biogeography, v. 33, n. 10, p. 1704-11, 2006. http://dx.doi.org/10.1111/j.13652699.2006.01460.x

PETERSON, A. T. Predicting species' geographic distributions based on ecological niche modeling. The Condor, v. 103, n. 3, p. 599-605, 2001.

http://dx.doi.org/10.1650/0010-5422(2001)103[0599:PSGDBO]2.0.CO;2

PETERSON, A.T.; BALL, L. G.; COHOON, K. P. Predicting distributions of Mexican birds using ecological niche modeling methods. Ibis, v. 144, n. 1, p. 27-32, 2002 a. http://dx.doi.org/10.1046/j.0019-1019.2001.00031.x

PETERSON, A. T.; ORTEGA-HUERTA, M. A.; BARTLEY, J.; SANCHEZ-CORDERO, V.; SOBERÓN, J.; BUDDEMEIER, R. H. et al Future projections for Mexican faunas under global climate change scenarios. Nature, v. 416, p. 626-29, 2002 b.

http://dx.doi.org/10.1038/416626a 
IBARRA-MONTOYA, J. L.; RANGEL-PERAZA, G.; GONZÁLEZ-FARIAS, F. A.; ANDA, J.; MARTÍNEZMEYER, E.; MACIAS-CUELLAR, H. M. Uso del modelado de nicho ecológico como una herramienta para predecir la distribución potencial de Microcystis sp (cianobacteria) en la Presa Hidroeléctrica de Aguamilpa, Nayarit, México. Ambi-Agua, Taubaté, v. 7, n. 1, p. 218-234, 2012. (http://dx.doi.org/10.4136/ambiagua.607)

PHILIPS, E. J.; ALDRIDGE, F. J.; HANSEN, P. Spatial and temporal variability of trophic state parameters in a shallow subtropical lake (Lake Okeechobee, Florida, USA). Archiv für Hydrobiology, v.128, p. 437-58, 1993.

PHILLIPS, S. J.; ANDERSON, R. P.; SCHAPIRE, R. E. Maximum entropy modeling of species geographic distributions. Ecological Modelling, v. 190, n. 3/4, p. 231-59, 2006. http://dx.doi.org/10.1016/j.ecolmodel.2005.03.026

PHILLIPS, S. J.; DUDIK, M. Modeling of species distributions with Maxent: new extensions and a comprehensive evaluation. Ecography, v. 31, n. 2, p. 161-75, 2008. http://dx.doi.org/10.1111/j.0906-7590.2008.5203.x

RANGEL-PERAZA, J. G.; DE ANDA, J.; GONZALEZ-FARIAS, F.; ERICKSON, D. Statistical assessment of water quality seasonality in large tropical reservoirs. Lakes \& Reservoirs: Research and Management, v. 14, n. 4, p. 315-23, 2009. http://dx.doi.org/10.1111/j.1440-1770.2009.00412.x

REYNOLDS, C. S. Cyanobacterial water blooms. In: CALLOW, P. (Ed.). Advances in botanical research. 13. ed. London: Academic Press, 1999. 143p. ISBN 0-419-23930-8.

ROSET, J.; AGUAYO, S.; MUÑOZ, M. J. Detección de cianobacterias y sus toxinas: una revisión. Revista de Toxicología, v. 18, n. 2, p. 65-71, 2001.

SOBERÓN, J.; PETERSON, A. T. Interpretation of models of fundamental ecological niches and species' distributional areas. Biodiversity Informatics, v. 2, p. 1-10, 2005.

SHAPIRO, J. Blue-green algae: why they become dominant. Science, v. 179, n. 4071, p. 38284, 1973. http://dx.doi.org/10.1126/science.179.4071.382

SOKAL, R. R.; ROHLF, F. J. Biometry: the principles and practice of statistics in biological research. New York: State University of New York; Stony Brook, 1995. 887 p. ISBN 0-7167-2411-1

WALSBY, A. E. Mechanisms of buoyancy regulation by planktonic cyanobacteria with gas esicles. In: FAY, P.; VAN BAALEN, C. (Ed.). The cyanobacteria. Amsterdan: Elsevier, 1997. 414 p.

WILEY, E. O.; MCNYSET, K. M.; PETERSON, A. T.; ROBINS, C. R.; STEWART, A. M. Niche modeling and geographic range predictions in the marine environment using a machine-learning algorithm. Oceanography, v. 16, n. 3, p. 120-7, 2003.

http://dx.doi.org/10.5670/oceanog.2003.42

ZAMBRANO, L.; MARTINEZ-MEYER, E.; MENEZES, N.; PETERSON, A. T. Invasive potential of common carp (Cyprinus carpio) and Nile tilapia (Oreochromis niloticus) in American freshwater systems. Canadian Journal of Fisheries and Aquatic Sciences, v. 63 , n. 9, p. 1903-10, 2006. http://dx.doi.org/10.1139/f06-088

ZAMUDIO, R. M.; GONZÁLEZ-FARIAS, F. Informe de fitoplancton del embalse de Aguamilpa. Mérida: Centro de Investigación y Asistencia en Tecnología y Diseño del Estado de Jalisco, A.C., 2009. 